\title{
Multikinase inhibitors in the treatment of thyroid cancer: specific role of lenvatinib
}

\author{
This article was published in the following Dove Press journal: \\ Biologics:Targets and Therapy \\ I0 April 2014 \\ Number of times this article has been viewed
}

\section{Neda Stjepanovic \\ Jaume Capdevila}

Department of Medical Oncology, Vall d'Hebron University Hospital, Barcelona, Spain
Correspondence: Jaume Capdevila Medical Oncology Department, Vall d'Hebron University Hospital, P Vall d'Hebron II9-129, 08035 Barcelona, Spain

Tel +349 32746085

Fax +34932746059

Email jacapdevila@vhebron.net

\begin{abstract}
Thyroid cancers are the most frequent neoplasms of the endocrine system and in the initial stages their prognosis is excellent. However, few therapeutic options are available for advanced or metastatic disease. In the last decade, a better understanding of the molecular events involved in the tumorigenesis of thyroid cancers has led to development of new targeted agents for the management of advanced and refractory disease. Multikinase inhibitors that are able to block pathways involved in the proliferation, invasion, and neoangiogenesis of thyroid cancer have been the most widely studied. After an international effort to identify and recruit sufficient patients, four placebo-controlled studies of multikinase inhibitors have been completed. These trials have led to the approval of the first agents with activity in advanced medullary thyroid cancers, which will probably change the landscape of treatment for iodinerefractory differentiated thyroid cancer in the near future. The purpose of this paper is to review the development of targeted agents for thyroid malignancy, with a special focus on lenvatinib, a multikinase inhibitor.
\end{abstract}

Keywords: thyroid cancer, lenvatinib, tyrosine kinase inhibitors, multikinase inhibitors, targeted therapies

\section{Introduction}

Thyroid cancer is the most prevalent endocrine malignancy and its incidence has been steadily increasing over the last three decades. ${ }^{1}$ This increase in incidence rate could reflect either increased detection or a true increase due to environment and lifestyle changes. ${ }^{2}$ Compared with other regions in the world, the highest incidence of thyroid cancer occurs in the female population of Northern America, which has an age-standardized rate of 15.1 per 100,000 females, while in Western Europe the age-standardized rate is 5.8 per 100,000 females. $^{3}$

Histopathologically, thyroid cancers are classified into three groups, ie, differentiated thyroid carcinoma (DTC) representing 94\% of all thyroid cancers and including the three subtypes of papillary (80\%), follicular (11\%) and Hurthle cell carcinoma (3\%), with medullary thyroid carcinoma (MTC) representing 4\% and anaplastic (undifferentiated) thyroid carcinoma (ATC) representing the remaining $2 \% .{ }^{4}$

The prognosis is excellent for the majority of patients treated by surgery, thyroidstimulating hormone-suppressive therapy, and radioiodine ablation in the case of DTC, with an overall survival rate of $97.7 \%$ at 5 years. ${ }^{5}$ Nevertheless, local recurrence occurs in up to $20 \%$ of patients, and distant metastases in approximately $10 \%$ at 10 years. $^{6}$ The natural evolution of relapsed thyroid cancer is towards metastatic dissemination and loss of ability to take up iodine, leading to a lack of response to 
the afore-mentioned standard therapies and an overall survival rate of less than $15 \%$ at 10 years. $^{7}$ The only approved systemic cytotoxic therapy for DTC is doxorubicin, which has a low response rate and an unfavorable toxicity profile, ${ }^{8}$ and no targeted treatments have been approved as yet for this type of thyroid cancer. Patients with advanced MTC derive limited benefit from cytotoxic drugs; however, in the last 3 years, due to enormous efforts and international collaboration, two targeted treatments have been approved for this type of thyroid cancer, ie, vandetanib ${ }^{9}$ and more recently cabozantinib. $^{10}$

Because of the lack of efficacy of traditional cytotoxic agents, novel treatments are needed for patients with thyroid cancer, especially for radioiodine-refractory and metastatic DTC. For the development of such new drugs, knowledge of the molecular pathways involved in the carcinogenesis of thyroid tumors is crucial.

Among the new therapeutic approaches for patients with advanced DTC, the most promising are the multikinase inhibitors, such as sorafenib, sunitinib, axitinib, vandetanib, pazopanib, and lenvatinib. Other novel treatments being evaluated include histone deacetylase inhibitors (vorinostat and depsipeptide), a DNS methylation inhibitor (decitabine), a heat-shock protein 90 inhibitor (17-AAG), a proteasome inhibitor (bortezomib), a selective cyclooxygenase-2 inhibitor (celecoxib), a derivative of thalidomide (lenalidomide), and mammalian target of rapamycin (mTOR) inhibitors (such as everolimus alone or in combination with multikinase inhibitors). ${ }^{4}$

This review focuses on multikinase inhibitors and the specific role of lenvatinib in the treatment of advanced thyroid cancer.

\section{Tyrosine kinases and molecular pathways in thyroid carcinoma}

Tyrosine kinases are enzymes that carry out protein phosphorylation, an important mechanism in cellular signal transduction in pathways that control cell proliferation, differentiation, migration, metabolism, and apoptosis. Tyrosine kinases are classified into two groups, ie, tyrosine kinase receptors (TKR), that are proteins located at the cell membrane and transduct extracellular signals to the cytoplasm when activated by ligands, ${ }^{11}$ and cellular tyrosine kinases that relay intracellular signals.

In cancer cells, constitutive activation of a pathway that leads to continuous oncogenic signaling can be a result of somatic or germline genetic alterations that cause overexpression or mutation of tyrosine kinases or other intracellular signals. Thyroid tumors frequently have genetic alterations leading to activation of the RET/PTC-RAS-RAFMAPK axis and phosphatidylinositol-3-kinase (PI3K)-AKTmTOR pathway (Figure 1).

The proto-oncogene RET (rearranged during transfection) encodes a membrane TKR, ${ }^{12}$ so rearrangements in the $R E T$ gene (RET/PTC) can lead to oncogenic activation of the mitogen-activated protein kinase (MAPK) pathway and development of cancer. Distally on the same pathway, activating mutations in RAS and RAF protein kinases can also have the same result. ${ }^{13}$ More than $70 \%$ of papillary thyroid carcinomas (PTC) harbor point mutations of $B R A F$ and $R A S$ genes $(40 \%-50 \%$ and $10 \%$, respectively) and RET/PTC rearrangement $(30 \%-40 \%)$, alterations that rarely overlap in the same tumor. ${ }^{14,15}$

The most frequent alterations found in follicular thyroid carcinomas (FTC) are $R A S$ mutations (40\%-50\%) and paired box 8/peroxisome proliferator-activated receptor gamma (PAX8/PPAR $\gamma$ ) rearrangements $(35 \%) .{ }^{12,16}$ PAX8/ PPAR $\gamma$ rearrangements result in a fusion oncogene that consists of the $P A X 8$ gene, a thyroid-specific transcription factor, and the PPAR $\gamma$ gene, a ubiquitously expressed transcription factor. This oncogene accelerates cell growth, reduces apoptosis, and permits independent growth of thyroid cell lines. ${ }^{17}$

Genetic alterations in PI3K-regulated intracellular signals and constitutive activation of the intracellular pathway downstream PI3K are frequent in more aggressive variants of thyroid cancer, such as poorly DTC and ATC. ${ }^{18}$ Pathway activation can be secondary to gene amplification of the serine-threonine-protein kinase AKT, activating mutations in the PI3K catalytic subunit (both more frequent in FTC and ATC than in PTC), or amplification of PIK3CA (16\% of PTC, $30 \%$ of FTC, and $50 \%$ of ATC). ${ }^{19}$

In familial MTC and the multiple endocrine neoplasia type 2 syndromes, a germline mutation causes activation of RET kinase ${ }^{20}$ and consequent activation of RAS, which leads to cross-activation of the MAPK and PI3K pathways. In sporadic MTC, about $50 \%$ of cases have amplified RASRAF signaling, leading also to cross-activation of the MAPK and PI3K pathway. ${ }^{21}$

RET is the most studied targetable TKR involved in the tumorigenesis of thyroid carcinoma, and is known to be able to activate a variety of signaling cascades, including MAPK and PI3K-AKT. Other TKR capable of activating the same pathways have been described, and include epidermal growth factor receptor, ${ }^{22}$ the vascular endothelial growth factor receptor (VEGFR) family, ${ }^{23}$ the fibroblast growth factor receptor 


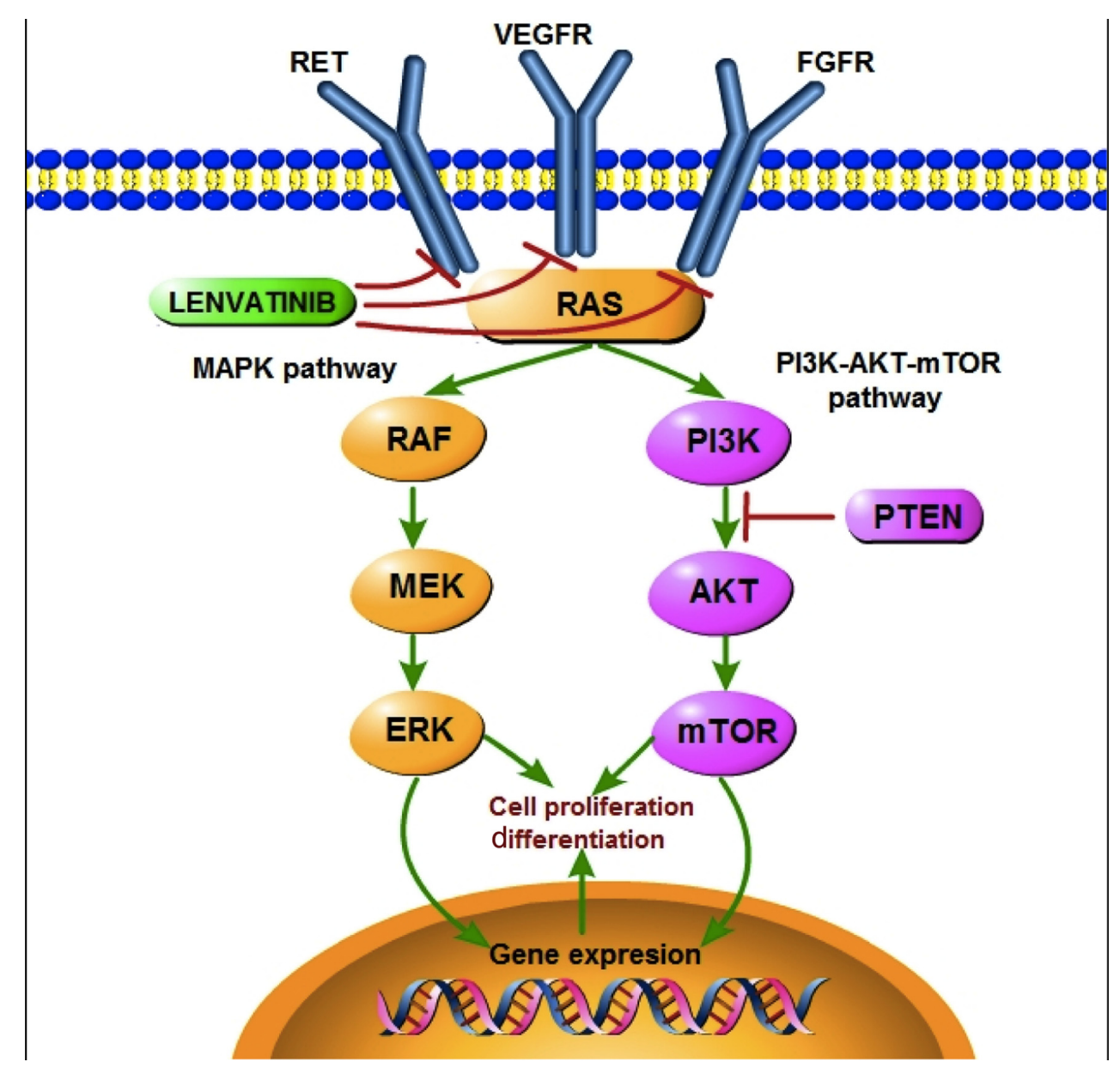

Figure I Kinase signaling cascade involved in development of thyroid carcinomas and representing the two main pathways, MAPK and PI3K-AKT-mTOR. Abbreviations: RET, rearranged during transfection tyrosine kinase receptor; VEGFR, vascular endothelial growth factor receptor; FGFR, fibroblast growth factor receptor; RAS, rat sarcoma protein; RAF, rapidly accelerated fibrosarcoma kinase; MEK or MAP2K, mitogen-activated protein kinase kinase: ERK or MAPK, mitogen-activated protein kinase; PI3K, phosphatidylinositide 3-kinase; PTEN, phosphatase and tensin homolog protein; AKT, protein kinase B; mTOR, mammalian target of rapamycin.

(FGFR) family, and the hepatocyte growth factor receptor encoded by the c-met (MET) proto-oncogene. ${ }^{24}$

The VEGFR family has three members, ie, VEGFR-1, VEGFR-2, and VEGFR-3, and their increased expression is characteristic of DTC. ${ }^{25,26}$ The VEGFR family is associated with increased tumor growth, progression, and invasiveness, and with decreased recurrence-free survival. VEGFR-2 is the most important mediator of tumor angiogenesis, ${ }^{27}$ while VEGFR-3 is expressed mainly in lymphatic endothelial cells, and is thought to be primarily involved in lymphangiogenesis.

The FGFR family has four members, ie, FGFR-1, FGFR-2, FGFR-3, and FGFR-4, which are well known to be expressed in thyroid cancers. ${ }^{28}$ The principal functions of FGFR are regulation of cell growth, proliferation, differentiation, and survival. ${ }^{29,30}$ In thyroid cancers, expression of FGFR-2 is diminished, indicating its protective role, and the rest of the receptors are overexpressed. While FGFR-1 and FGFR-3 are overexpressed in well differentiated tumor types, FGFR-4 is overexpressed in more aggressive tumors, suggesting its role in tumor progression. ${ }^{31}$

\section{Tyrosine kinase inhibitors and resistance}

Thyroid cancers with constitutive activation in a pathway secondary to TKR alteration can be sensitive to two types of specific inhibitors of the implied tyrosine kinase, ie, monoclonal antibodies and small molecule tyrosine kinase inhibitors (TKIs).

The benefits of TKR inhibitors can be inexistent or transient, due to distinct mechanisms of drugs resistance followed by tumor progression. Two modes of resistance have been described for the VEGF pathway inhibitors: intrinsic, manifesting no benefit from the drug, and evasive, reflecting the capacity of the tumor to avoid blockade of angiogenesis. Intrinsic resistance may be explained by the pre-existing tumor microenvironment that confers 
the indifference to the drug. Evasive resistance involves numerous mechanisms such as: upregulation of alternative proangiogenic signals leading to revascularization; recruitment of proangiogenic inflammatory cells or pericyte coverage providing protection for the tumor vasculature; local tissue invasion acquiring vascularization from normal vasculature; and, finally, metastasis to lymph nodes and distant organs. ${ }^{32}$

Among the alternative proangiogenic signals, upregulation of FGF/FGFRs has been described. Recent data from preclinical studies suggest that the FGFR pathway is a driver of resistance to antiangiogenic drugs, so anti-FGF/FGFR agents can be used to reverse acquired resistance to antiVEGFR or other targeted agents. ${ }^{33}$

Considering that most solid tumors show deregulation of multiple signaling pathways and upregulation of pathways as a mechanism of resistance, combinations of various TKIs with different targets have been investigated in preclinical and clinical studies with positive results. For these reasons, current drug development tends to focus on creating a single drug capable of targeting several tyrosine kinases and oncogenic pathways simultaneously, ie, a multikinase inhibitor.

\section{Multikinase inhibitors in thyroid carcinoma}

Recent studies of multikinase inhibitors in advanced thyroid carcinoma have shown prolonged tumor response and clinical benefit (Table 1), causing a shift in the treatment of this group of patients. The effectiveness of these drugs can be explained by their ability to inhibit several pathways simultaneously (like PI3K and MAPK, the two most important pathways in thyroid carcinoma) and their role as inhibitors of angiogenesis. Several of these agents are currently under clinical investigation in patients with DTC and MTC, and some have already been approved for treatment of advanced MTC.

Motesanib diphosphate is an oral inhibitor of the tyrosine kinases of VEGFR-1, VEGFR-2, VEGFR-3, the plateletderived growth factor receptor (PDGFR), c-KIT, and wildtype RET. ${ }^{34}$ Two Phase II trials using motesanib diphosphate $125 \mathrm{mg}$ once daily have been conducted in patients with advanced or metastatic thyroid cancer. The first trial included

Table I Main studies in advanced thyroid cancer with targeted therapies.

\begin{tabular}{|c|c|c|c|c|c|c|c|}
\hline Reference & Drug & Phase & $\mathbf{N}$ & Tumor type & $\mathbf{R R} \%$ & SD\% & $\begin{array}{l}\text { mPFS, } \\
\text { months }\end{array}$ \\
\hline Sherman et al, $2008^{35}$ & Motesanib & 2 & 93 & DTC & 14 & $35^{a}$ & 9.2 \\
\hline Schlumberger et al, $2009^{36}$ & Motesanib & 2 & 91 & MTC & 2 & $48^{\mathrm{a}}$ & II \\
\hline Cohen et al, $2008^{37}$ & Axitinib & 2 & 60 & All & 30 & 38 & 18.1 \\
\hline Locati et al, $2012^{38}$ & Axitinib & 2 & 52 & DTC, MTC & 34.6 & 34.6 & NR \\
\hline Kloos et al, $2009^{39}$ & Sorafenib & 2 & 41 & PTC & 15 & $56^{\mathrm{a}}$ & 16 \\
\hline Gupta-Abramson et al, $2008^{40}$ & Sorafenib & 2 & 30 & DTC & 23 & 53 & 19.3 \\
\hline Hoftijzer et al, $2009^{41}$ & Sorafenib & 2 & 31 & DTC & 25 & 34 & 14.5 \\
\hline Ahmed et al, $201 \mathrm{I}^{42}$ & Sorafenib & 2 & 26 & DTC, MTC & 18 & 82 & NR \\
\hline Brose $M$ et al, $2013^{43}$ & Sorafenib vs. Placebo & 3 & 417 & DTC & I 2.2 vs. 0.5 & $42 v s .33^{a}$ & 10.8 vs. 5.8 \\
\hline Lam et al, $2010^{44}$ & Sorafenib & 2 & 15 & $\mathrm{MTC}^{\mathrm{b}}$ & 6 & 88 & 17.9 \\
\hline \multirow[t]{3}{*}{ Capdevila et al, $2012^{45}$} & Sorafenib & $\mathrm{R}$ & 34 & MTC & 47 & 40 & 10.5 \\
\hline & & & & DTC & 19 & 50 & 13.5 \\
\hline & & & & ATC & 33 & 0 & 4.4 \\
\hline Cohen EE et al, $2008^{46}$ & Sunitinib & 2 & 43 & DTC, MTC & $13^{d}$ & $68^{d}$ & NR \\
\hline Ravaud et al ,200847 & Sunitinib & 2 & 17 & All & 6 & 80 & NR \\
\hline \multirow[t]{2}{*}{ Carr et al, $2010^{48}$} & Sunitinib ${ }^{e}$ & 2 & 35 & DTC, & 31 & 46 & 12.8 \\
\hline & & & & MTC & & & \\
\hline de Souza et al, $2010^{49}$ & Sunitinib & 2 & 25 & MTC & 35 & 57 & 7 \\
\hline Bible et al, $2010^{50}$ & Pazopanib & 2 & 39 & DTC & 49 & NR & 11.7 \\
\hline Bible et al, $2012^{51}$ & Pazopanib & 2 & 16 & ATC & 0 & NR & 2 \\
\hline Wells et al, $2010^{52}$ & Vandetanib & 2 & 30 & $M^{\prime} C^{c}$ & 20 & 53 & 27.9 \\
\hline Robinson et al, $2010^{53}$ & Vandetanib & 2 & 19 & $M^{\prime} C^{c}$ & 16 & $53^{a}$ & NR \\
\hline Wells et al, $2012^{9}$ & Vandetanib vs. Placebo & 3 & 331 & MTC & 45 vs. 13 & NR & 30.5 vs. 19.3 \\
\hline Leboulleux et al, $2012^{54}$ & Vandetanib vs. Placebo & 2 & 145 & DTC & 8.3 & NR & II,I vs. 5,9 \\
\hline Kurzrock et al, 201 I55 & Cabozantinib & 1 & 37 & MTC & 29 & 41 & NR \\
\hline Elisei et al, $2013^{10}$ & Cabozantinib vs. Placebo & 3 & 330 & MTC & 28 vs. 0 & NR & II .2 vs. 4 \\
\hline
\end{tabular}

Abbreviations: NR, not reported or not reached; R, retrospective study; a: >6 m; b: sporadic; c: hereditary; d: results given for the DTC group; e: patients with 2-deoxy2-[18F]fluoro-D-glucose positron emission tomography-avid thyroid cancers. 
93 patients with advanced radioiodine-resistant DTC. The objective response rate was $14 \%$, and stable disease was maintained for 24 weeks or longer in 35\% of patients. Among those who progressed, median progression-free survival was 40 weeks. Serum thyroglobulin levels decreased by more than $50 \%$ in $45 \%$ of patients (in $15 \%$ beyond 24 weeks), and this biomarker response correlated with radiological response. ${ }^{35}$ The second trial included 91 patients with advanced MTC, with a partial response achieved in $2 \%$, stable disease beyond 24 weeks in $48 \%$, and a median progression-free survival of 48 weeks. A decrease in plasma calcitonin and carcinoembryonic antigen levels was seen in $83 \%$ and $75 \%$ of patients, respectively. ${ }^{36}$ Treatment-related adverse events in both trials included diarrhea, fatigue, hypertension, and anorexia.

Axitinib is another oral inhibitor of VEGFR-1, VEGFR-2, VEGFR-3, PDGFR, and c-KIT, but does not target RET. A Phase II trial using axitinib $5 \mathrm{mg}$ twice daily was done in 60 patients with advanced or metastatic thyroid cancer. A partial response was achieved in all histological subtypes, with an objective response rate of $30 \%$, stable disease lasting more than 16 weeks in $38 \%$, and a median progression-free survival of 18.1 months. The effect on serum thyroglobulin concentrations was evaluated in 21 patients, with a decrease noted in most cases, regardless of radiological response. Calcitonin levels were measured in seven patients, and a significant reduction was noted only in the two patients with a partial response. The most common adverse effect was hypertension. ${ }^{37}$ Another Phase II trial used axitinib $7 \mathrm{mg}$ twice daily initially and a maximum dose of $10 \mathrm{mg}$ if well tolerated. This trial recruited 52 patients with advanced iodine-refractory DTC or MTC, with a partial response achieved in $34.6 \%$ and stable disease in $34.6 \%$. The two main adverse events were hypertension and fatigue. ${ }^{38}$

Sorafenib is an oral inhibitor of VEGFR-1, VEGFR-2, VEGFR-3, PDGFR, RET, c-KIT, and RAF (C-Raf and $\mathrm{B}-\mathrm{Raf}$ ). This drug has been investigated previously in four prospective Phase II trials, ${ }^{39-42}$ and shown promising activity in terms of response rate $(15 \%-25 \%)$ and median progression-free survival (14-20 months, Table 1). These results led to the design of a pivotal, double-blind, Phase III study known as DECISION, that examined the efficacy and safety of sorafenib $400 \mathrm{mg}$ twice daily versus placebo in 417 patients with progressive radioiodine-refractory DTC. The primary endpoint was met, with significant improvement in median progression-free survival for sorafenib versus placebo (10.8 months versus 5.8 months, hazards ratio $0.587, P<0.0001$ ), maintaining the benefit of sorafenib across different subgroups (eg, regions, age). The objective response rate (including all partial responses) in the sorafenib arm versus the placebo arm was $12.2 \%$ versus $0.5 \%$, respectively, the stable disease rate for more than 6 months was $42 \%$ versus $33 \%$, with a disease control rate of $54.1 \%$ versus $33.8 \%(P<0.0001)$. Although the independent central review confirmed a $12 \%$ objective response rate by RECIST (Response Evaluation Criteria In Solid Tumors) criteria, most patients (73\%) showed some degree of reduction in target tumor lesions. Median overall survival has not been reached, and at the time of analysis, these data are probably affected by the fact that the majority of patients from the placebo group have already or will crossover to the sorafenib group. The most common adverse events in the sorafenib arm included hand-foot syndrome, diarrhea, alopecia, rash, fatigue, weight loss, and hypertension. Dose modifications due to adverse events were required in $77.8 \%$ and $30.1 \%$ of patients in the sorafenib and placebo groups, respectively. Permanent discontinuation due to adverse events was also more common in the sorafenib group (18.8\%) than in the placebo group $(3.8 \%){ }^{43}$

Sorafenib was also investigated in a Phase II study of 16 patients with sporadic metastatic MTC. A parallel arm of patients with hereditary MTC was terminated prematurely because of slow patient enrollment. ${ }^{44} \mathrm{~A}$ partial response was seen in only one patient $(6.2 \%)$, but stable disease beyond 15 months was seen in $50 \%$ of patients. Calcitonin and carcinoembryonic antigen levels were measured in 20 patients, with $85 \%$ showing a decrease in levels of either marker, but no correlation with radiological response was observed. Adverse events were generally consistent with the known profile of sorafenib. After 12 months of treatment, patients with MTC from a UK study ${ }^{42}$ had a radiological objective response rate of $25 \%$ and overall survival of $100 \%$. In a retrospective Spanish analysis of 34 patients with advanced and refractory thyroid cancer treated using sorafenib, the objective response rate was $47 \%$ for MTC, $19 \%$ for DTC, and $33 \%$ for ATC. With a median follow-up of 11.5 months, median progression-free survival was $13.5,10.5$, and 4.4 months for DTC, MTC, and ATC respectively. ${ }^{45}$

Sunitinib targets PDGFR, VEGFR, c-Kit, RET, macrophage colony-stimulating factor 1 receptor, and the FL cytokine receptor, FLT3. Most of the Phase II trials evaluating sunitinib in advanced thyroid cancer have used daily doses of $50 \mathrm{mg}$ on a 4 -week on/2-week off schedule (Table 1). Three of the trials ${ }^{46-48}$ included patients with DTC and MTC, and reported objective response rates of $6 \%-31 \%$ and stable disease in $46 \%-80 \%$ of patients. Median progression-free survival was reported in only one of the studies, and was 
12.8 months. ${ }^{48}$ Another study that included only patients with MTC reported an objective response rate of $35 \%$, with stable disease in $57 \%$ of patients and a median progression-free survival of 28 weeks. In this study, RET-activating mutations were found in $85 \%$ of the tumors analyzed, and this group was shown to have more durable responses, with a one-year probability of progression-free survival of $88 \%{ }^{49}$ The most common treatment-related adverse events were similar in all the trials, and included neutropenia, thrombocytopenia, lymphopenia, hypertension, hand-foot syndrome, fatigue, and gastrointestinal symptoms.

Pazopanib targets the VEGFR family, PDGF, and c-Kit, and was studied at a dose of $800 \mathrm{mg}$ daily in a Phase II study of 39 patients with progressive, radioiodine-resistant DTC. A partial response was reported in $49 \%$ of patients and median progression-free survival was 11.7 months. Thyroglobulin concentrations decreased by at least $30 \%$ from baseline levels in $88 \%$ of 32 patients assessed. The most frequent all-grade adverse events reported were fatigue, changes in skin and hair pigmentation, diarrhea, and nausea. ${ }^{50}$ Another Phase II trial was conducted in 16 patients with ATC who were treated with pazopanib $800 \mathrm{mg}$ daily, and found no confirmed RECIST responses, with a median time to progression of 62 days and a median survival time of 111 days. $^{51}$

Vandetanib effectively blocks RET tyrosine kinase, as well as VEGFR-1, VEGFR-2, and the epidermal growth factor receptor. Vandetanib has been assessed at different doses in Phase II and III clinical trials. Two Phase II trials ${ }^{52,53}$ investigated vandetanib in patients with hereditary MTC, and showed objective response rates of $16 \%-20 \%$, stable disease in $53 \%$ of patients, and a median progression-free survival of 27.9 months in the first study using a dose of $300 \mathrm{mg}$. These results led to a Phase III trial in patients with advanced hereditary or sporadic MTC using $300 \mathrm{mg}$ of vandetanib daily versus placebo. This study recruited 331 patients, with the results showing a significantly prolonged progression-free survival on vandetanib compared with placebo (19.3 months in placebo group versus not reached in vandetanib group, although a predicted median progression-free survival of 30.5 months was obtained using Weibull model, HR: 0.46; 95\% CI, 0.31 to $0.69 ; P<0.001)$ and a significantly major objective response rate of $45 \%$ and $1 \%$, respectively. RET mutation status was positive in $56.5 \%$ of patients, negative in $2.4 \%$, and unknown in $41.1 \%$, indicating no correlation between RET mutation status and clinical outcome. ${ }^{9}$ The results of this Phase III study led to the US Food and Drug Administration and the European Medicines Agency approving vandetanib as the first systemic treatment for metastatic or progressive MTC in adult patients.

A Phase II trial of vandetanib versus placebo was conducted in 145 patients with advanced DTC. Patients in the vandetanib group had longer progression-free survival than those in the placebo group, with a median progression-free survival of 11.1 months versus 5.9 months, respectively (hazards ratio 0.63). ${ }^{54}$ Currently, a double-blind, placebo-controlled Phase III study (VERIFY, NCT01876784) is evaluating the efficacy of vandetanib in advanced, radioiodine-refractory DTC. The most common adverse events for vandetanib in all the trials were clinically insignificant QT interval corrected for heart rate prolongation, diarrhea, rash, nausea, hypertension, asthenia, and fatigue.

Cabozantinib inhibits the hepatocyte growth factor receptor, VEGFR-2, and RET. The clinical benefit of cabozantinib for patients with advanced MTC was observed early in a Phase I study. Its promising clinical activity led to expansion of an MTC-enriched cohort to include 37 patients with MTC, representing 44\% of the total study population. In the MTC cohort, a partial response was observed in $29 \%$ of patients, along with stable disease for at least 6 months in $41 \%$. The maximum tolerated dose was $175 \mathrm{mg}$ daily, and grade 3 adverse effects comprised hand-foot syndrome and mucositis, along with elevated aspartate aminotransferase, alanine aminotransferase, and lipase. ${ }^{55}$

Following the impressive results of the Phase I study, a Phase III study of cabozantinib $140 \mathrm{mg}$ daily was conducted directly in 330 patients with metastatic MTC. Median progression-free survival was 11.2 months for cabozantinib and 4 months for placebo, with benefit observed across all subgroups according to age, prior TKI treatment, and RET mutation status. The objective response rate was $28 \%$ for cabozantinib and $0 \%$ for placebo, regardless of RET mutation status. Progression-free survival at one year was $47.3 \%$ for cabozantinib and $7.2 \%$ for placebo. Common adverse events associated with cabozantinib included diarrhea, handfoot syndrome, decreased weight, nausea, and fatigue, along with a need for dose reduction in $79 \%$ of patients and treatment discontinuation in $16 \% .{ }^{10}$ The results of the Phase III trial led to US Food and Drug Administration approval of a second multikinase inhibitor for patients with progressive metastatic MTC.

\section{Lenvatinib}

Lenvatinib (E7080) is an oral multiple RTK inhibitor targeting VEGFR-1-3, FGFR-1-4, RET, c-KIT, and PDGFR $\beta^{56}$ (Figure 2). Lenvatinib is presently under investigation both 

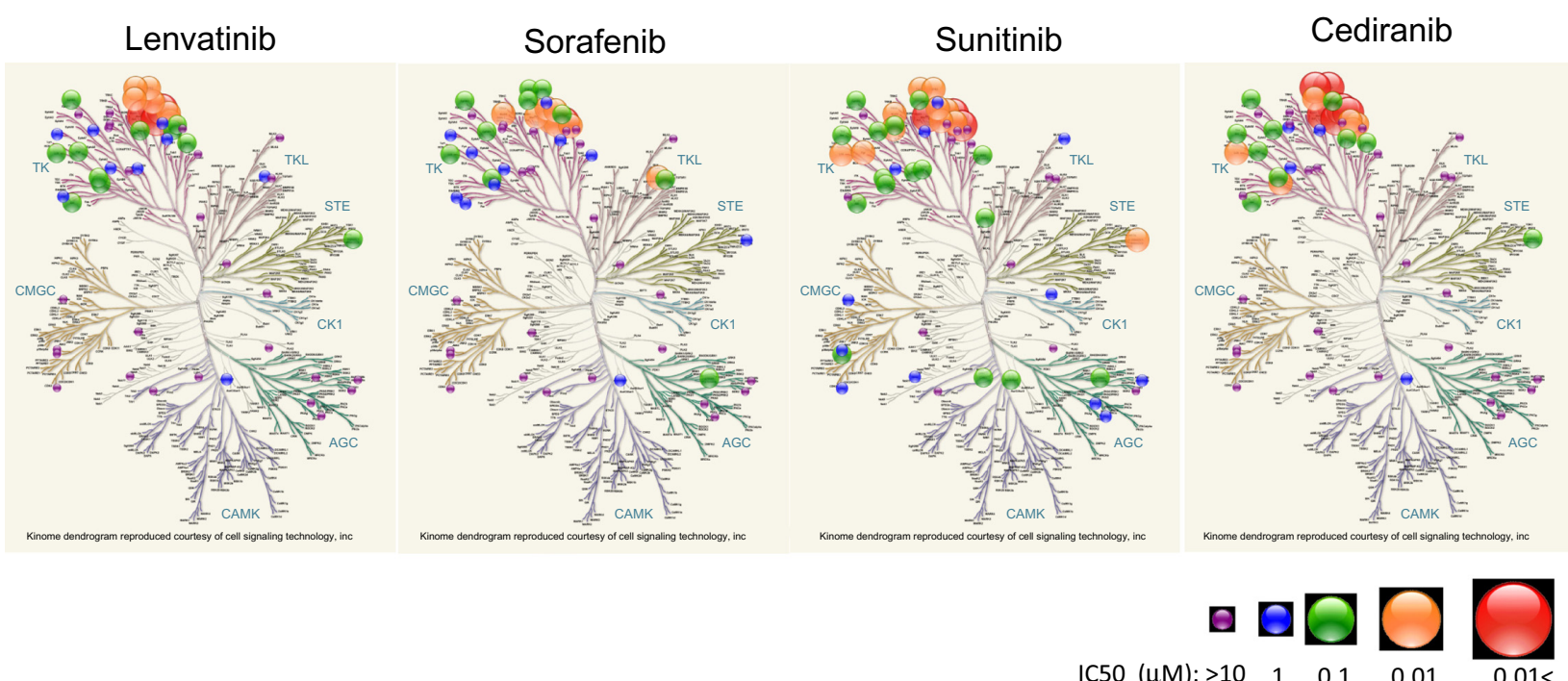

IC50 $(\mu \mathrm{M}):>10$

Figure 2 Kinome analysis of lenvatinib. Tyrosine kinase assays were performed by an ELISA and Off-chip Mobility Shift Assay (MSA) by Carna Biosciences, Inc. (Kobe, Japan). A kinome map was made using Cell Signaling Technology (Beverly, MA) methodology. Illustration reproduced courtesy of Cell Signaling Technology, Inc. (www.cellsignal.com).

as monotherapy and in combination with cytotoxic agents for multiple types of solid tumor, including thyroid carcinoma and hepatocellular carcinoma (in Phase III trials), and melanoma, renal carcinoma, non-small cell lung carcinoma, glioblastoma multiforme, and ovarian and endometrial carcinoma (Phase I and II trials).

The rationale for development of lenvatinib as an antitumor drug lies in its ability to interfere with angiogenesis, one of the main processes involved in survival, local invasion, and metastasis of tumor cells. Lenvatinib targets three of the many extracellular and intracellular molecules capable of modulating angiogenesis and lymphangiogenesis, ie, VEGFR, FGFR, and PDGFR $\beta$. The difference between lenvatinib and other TKIs with antiangiogenesis properties is its potency with regard to inhibition of FGFR-1, ${ }^{56,57}$ offering a potential opportunity to block one of the well known mechanisms of resistance to VEGF/VEGFR inhibitors. Lenvatinib also has a direct oncogenic effect of controlling tumor cell proliferation by inhibiting RET, c-KIT, and PDGFR beta, and an effect on the tumor microenvironment by blocking FGFR and PDGFR beta (Figure 3 and Table 2).

\section{Phase I trial}

The safety, tolerability, and antitumor efficacy of lenvatinib were assessed in a Phase I trial that included 82 patients with advanced, refractory solid tumors (12 colorectal carcinoma, 18 sarcoma, 15 melanoma, eight renal carcinoma, six gastric carcinoma, four pancreatic carcinoma, four mesothelioma, four pancreatic carcinoma, three ovarian

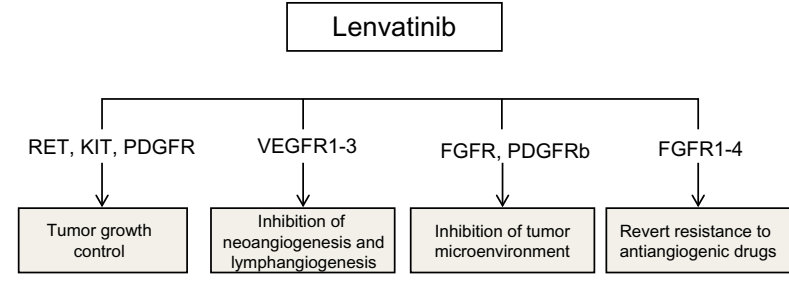

Figure 3 Proposed mechanism of action of lenvatinib.

Abbreviations: RET, rearranged during transfection tyrosine kinase receptor; VEGFR, vascular endothelial growth factor receptor; FGFR, fibroblast growth factor receptor; PDGFR, platelet-derived growth factor receptor.

carcinoma, three esophageal carcinoma, and others). ${ }^{58}$ Lenvatinib was administered orally in escalating doses from $0.2 \mathrm{mg}$ to $32 \mathrm{mg}$ daily in 28-day cycles. The dose-limiting toxicity was grade 3 proteinuria, observed in two patients at $32 \mathrm{mg}$, and the maximum tolerated dose was defined as $25 \mathrm{mg}$. The most frequent drug-related toxicities were hypertension ( $40 \%$ of patients), diarrhea (45\%), nausea $(37 \%)$, stomatitis $(32 \%)$, proteinuria $(26 \%)$ and vomiting $(23 \%)$. Dose modifications were required in $49 \%$ of patients (most occurring at doses of $12 \mathrm{mg}$ or higher). At the maximum tolerated dose of $25 \mathrm{mg}$, dose modifications were required in $54 \%$ of patients, most commonly due to hypertension and proteinuria. A partial response in terms of antitumor activity was seen in $9 \%$ of patients (with renal cell carcinoma, melanoma, or soft tissue sarcoma), with stable disease in $46 \%$.

Pharmacokinetic investigation showed that lenvatinib is absorbed rapidly, reaching peak plasma concentrations in approximately 3 hours, with dose-linear kinetics and no drug 
Table 2 Profile of tyrosine kinase inhibitors in development ${ }^{57}$

\begin{tabular}{|c|c|c|c|c|c|c|c|c|}
\hline \multirow[t]{2}{*}{ TKR } & \multicolumn{8}{|c|}{$I C_{50}(\mathrm{nmol} / \mathrm{L})$} \\
\hline & Motesanib & Axitinib & Sorafenib & Sunitinib & Pazopanib & Vandetanib & Cabozantinib & Lenvatinib \\
\hline VEGFR-I & 2 & 0.1 & 26 & 10 & 10 & - & - & 22 \\
\hline VEGFR-2 & 3 & 0.2 & 90 & 10 & 30 & 40 & 0.035 & 4 \\
\hline VEGFR-3 & 6 & 0.29 & 20 & 10 & 47 & 110 & - & 5.2 \\
\hline PDGFR $\beta$ & 84 & 2 & 57 & 39 & 84 & - & - & 39 \\
\hline c-KIT & 8 & 1.7 & 68 & $1-10$ & 74 & - & - & - \\
\hline RET & 59 & 1.2 & 47 & 100 & - & 130 & 4 & 35 \\
\hline RET/PTC & - & - & 50 & 224 & - & 100 & - & - \\
\hline BRAF & - & - & 25 & - & - & - & - & - \\
\hline Others $\left(\mathrm{IC}_{50}\right)$ & - & - & - & - & - & EGFR (500) & c-MET (I.8) & FGFR-I (I.8) \\
\hline
\end{tabular}

Abbreviations: EGFR, epidermal growth factor receptor; FGFR, fibroblast growth factor receptor; VEGFR, vascular endothelial growth factor receptor; PDGFR, plateletderived growth factor receptor; TKR, tyrosine kinase receptor; RET, rearranged during transfection tyrosine kinase receptor.

accumulation after administration for 4 weeks. In a study of the effects of food in eleven patients, no changes in peak plasma levels were observed, but the time to peak plasma levels was longer in the fed group than in the fasted group (5 hours versus 2 hours, respectively). ${ }^{58}$

\section{Phase II trials}

On the basis of the Phase I experience and the rationale that expression of FGFR can contribute to control of neoangiogenesis in thyroid cancer, a multicenter, openlabel Phase II trial with two different cohorts was initiated. Between October 2008 and February 2010, 58 patients with radioiodine-refractory DTC (74\% PTC) were enrolled. All the patients were treated with lenvatinib $24 \mathrm{mg}$ daily. Thirty percent of the patients had received prior anti-VEGFR therapy. The median duration of treatment was 405 days, with a minimum follow-up of 14 months. A partial response was observed in 59\% and stable disease in 36\%, with a median progression-free survival of 13.3 months (comparable for patients with and without prior VEGFR TKI therapy). The most frequent treatment-related adverse effects were typical of this drug class, ie, hypertension, proteinuria, weight loss, diarrhea, and fatigue, leading to dose reductions in $39 \%$ of patients and drug withdrawal in $29 \%$. A decrease in thyroglobulin was observed during the second cycle and was correlated with maximum tumor shrinkage. $R A S$ mutations (NRAS and KRAS) were found in $35 \%$ of patients, with a $100 \%$ response rate observed in this group and a significantly improved median progression-free survival ( $80 \%$ at 14 months versus $20 \%$ in the wild-type RAS group). ${ }^{59}$

Circulating angiogenic factor levels were assessed at baseline and 8 days post treatment, with significant changes seen in 14 of the 50 circulating angiogenic factors assayed. Increased tumor shrinkage correlated with low baseline levels of angiopoietin-2, and the extent of tumor shrinkage increased with increasing fold changes in fibroblast growth factor-2 and interleukin-10 levels. A significant association was also observed between progression-free survival and low baseline VEGF alpha and angiopoietin-2 levels. Compared with wildtype individuals, patients with KRAS and NRAS mutations had significantly lower VEGF levels and significantly higher soluble Tie-2 levels, suggesting that inherent sensitivity of DTC cells to lenvatinib may be directly linked to KRAS/NRAS mutations, or indirectly through inhibition of VEGF alpha and angiopoietin- 2 by induction of soluble Tie- $2 .^{60}$

Fifty-nine patients were enrolled from October 2008 to August 2010 for the MTC cohort of this Phase II trial. The treatment schedule was the same as in the DTC cohort, and $44.1 \%$ of patients had previously received anti-VEGF agents. The median duration of treatment was 264 days. No difference in adverse effects was observed between the MTC and DTC cohorts, with $22 \%$ of the MTC cohort requiring discontinuation of treatment and dose reduction being needed in 58\%. At a minimum of 8 months' follow-up, $36 \%$ of patients had a partial response, $29 \%$ had durable stable disease (for more than 23 weeks), median progression-free survival was 9 months (12.9 months in patients without prior VEGF-targeted therapy versus 7.3 months in patients with prior VEGF-targeted therapy), and median overall survival was 16.6 months for the entire MTC study population. Biomarkers were assessed in 24 patients, and a RET mutation was found in $67 \%$ of patients; however, this had no correlation with objective response rate or progression-free survival. Clinical benefit in terms of disease control rate (partial response + stable disease) was observed in RET M918T mutant-positive patients, but was not statistically significant. Serum calcitonin and carcinoembryonic antigen levels decreased in all patients and were correlated with response. After the first cycle, a median reduction in calcitonin was documented in $65 \%$ of patients with a partial 
response compared with a $30 \%$ reduction in patients with progressive disease. Median carcinoembryonic antigen levels were $46 \%$ lower in patients with a partial response compared with a $9 \%$ decrease in patients with progressive disease. ${ }^{61}$

Six circulating angiogenic factors (placenta-derived growth factor, interferon gamma-induced protein 10, VEGF alpha, stromal cell-derived factor-1 alpha, granulocyte colony-stimulating factor, and eotaxin) increased significantly, whereas five circulating angiogenic factors (VEGFR-1-3, angiopoietin-2, and soluble Tie-2) decreased significantly 8 days post treatment. Low baseline levels of angiopoietin-2, soluble Tie-2, hepatocyte growth factor, and interleukin- 8 were associated with greater tumor shrinkage and Ang-2, interleukin-8, and hepatocyte growth factor were also associated with prolonged progression-free survival. High baseline levels of VEGF, PDGF-BB, and VEGFR-3 were associated with greater tumor shrinkage. ${ }^{61}$

\section{Phase III trial}

The SELECT trial (NCT01321554) is a multicenter, randomized, double-blind, placebo-controlled study of lenvatinib in radioiodine-refractory DTC patients. The study is planned to treat progressive DTC patients with lenvatinib $24 \mathrm{mg}$ daily or placebo in a randomization ratio of $2: 1$. The primary endpoints are progression-free survival, sec-

Table 3 Differences in study design between DECISION trial (sorafenib versus placebo) and SELECT trial (lenvatinib versus placebo) in patients with progressive radioactive iodine-refractory differentiated thyroid carcinoma

\begin{tabular}{|c|c|c|}
\hline & DECISION & SELECT \\
\hline Subjects & 417 & 360 \\
\hline Study period & 21 months & 36 months \\
\hline Randomization & $\mathrm{I}: \mathrm{I}$ & $2: 1$ \\
\hline Primary objective & PFS & PFS \\
\hline Secondary objectives & ORR, OS & ORR, OS \\
\hline Prior anti-VEGFR therapy & No & Yes (maximum one) \\
\hline $\begin{array}{l}\text { Evidence of disease } \\
\text { progression }\end{array}$ & Prior 14 months & Prior 12 months \\
\hline $\begin{array}{l}\text { Centrally confirmed } \\
\text { radiologic progression } \\
\text { prior to randomization }\end{array}$ & No & Yes \\
\hline Tumor evaluation & RECIST I.0 & RECIST I.I \\
\hline $\begin{array}{l}\text { Response evaluation by } \\
\text { independent central review }\end{array}$ & Yes & Yes \\
\hline $\begin{array}{l}\text { Crossover for placebo } \\
\text { patients }\end{array}$ & Yes & Yes \\
\hline
\end{tabular}

Note: Both are double-blind, randomized, multicenter Phase III trials.

Abbreviations: ORR, objective response rate; OS, overall survival; VEGFR, vascular endothelial growth factor receptor; PFS, progression-free survival; RECIST, Response Evaluation Criteria In Solid Tumors. ondary objective response rate, and overall survival, with an efficacy evaluation every 8 weeks. Approximately 392 patients have been enrolled in 23 countries (USA, Argentina, Australia, Austria, Belgium, Brazil, Canada, Chile, Czech Republic, Denmark, France, Germany, Italy, Japan, Korea, Poland, Portugal, Romania, Russia, Spain, Sweden, Thailand, and the UK) and recruitment was completed in October 2012.

Although the SELECT and DECISION trials tested TKIs in the same setting, there were slightly but significant differences between them that should be taken into account when interpreting the data. One of the most important differences is previous therapy, which was not allowed in the DECISION trial but was permitted in the SELECT trial, including one previous treatment with a VEGFR inhibitor. Another noteworthy difference is the centrally confirmed progressive disease prior to inclusion in the SELECT trial, whereas in the DECISION trial this prerequisite was assessed by the investigators (Table 3 ). These differences could influence progression-free survival and response rates in both arms, making it difficult to compare data between the trials. A recent press release has communicated that the SELECT trial has met the primary endpoint of progressionfree survival benefit in treatment of radioiodine-refractory DTC and lenvatinib has been submitted for market authorization worldwide.

\section{Conclusion}

The multikinase inhibitors represent a shift in the treatment of advanced thyroid cancers, and have achieved prolonged responses and improved progression-free survival. Several agents are being tested, with vandetanib and cabozantinib now approved for the treatment of advanced MTC. Sorafenib and lenvatinib are being assessed for advanced radioiodinerefractory DTC in Phase III clinical trials, with positive results emerging already from the sorafenib trial and recently also for lenvatinib pivotal study. In the near future, we will be faced with a number of treatment options for this patient population, with little evidence for which drug should be used first and whether or not there is crossover drug resistance. Further, in this group of patients, where no chance of cure is possible and TKIs have still not demonstrated a survival benefit, the adverse effects of long-lasting treatment with TKIs could worsen quality of life (which is mostly excellent before starting treatment with these agents). Taking these factors into account, we should be able to choose precisely which patients should be treated with novel targeted therapies. In order to achieve clinical benefit and preserve quality of 
life, patients should be better selected, and development of reliable biomarker programs running in parallel with drug development is urgently needed.

\section{Disclosure}

The authors report no potential conflicts of interest in this work.

\section{References}

1. Pellegriti G, Frasca F, Regalbuto C, Squatrito S, Vigneri R. Worldwide increasing incidence of thyroid cancer: update on epidemiology and risk factors. J Cancer Epidemiol. 2013;2013:965212.

2. Chen A, Jemal A, Ward EM. Increasing incidence of differentiated thyroid cancer in the United States,1988-2005. Cancer. 2009;115(16): 3801-3807.

3. Cancer Research UK org. Thyroid cancer incidence statistics. Available from: http://www.cancerresearchuk.org/cancer-info/cancerstats/types/ thyroid/incidence/uk-thyroid-cancer-incidence-statistics-geog. Accessed November 5, 2013.

4. National Comprehensive Cancer Network. Thyroid Carcinoma (version 2.2013). Available from: http://www.nccn.org/professionals/ physician_gls/pdf/thyroid.pdf. Accessed November 5, 2013.

5. National Cancer Institute. Surveillance Epidemiology and End Results Program: SEER Stat Facts: Thyroid Cancer. 2011. Available from: http:// seer.cancer.gov/statfacts/html/thyro.html-incidence-mortality. Accessed November 5, 2013.

6. Eustatia-Rutten CF, Corssmit EP, Biermasz NR, Pereira AM, Romijn JA, Smit JW. Survival and death causes in differentiated thyroid carcinoma. J Clin Endocrinol Metab. 2006;91(1):313-319.

7. Durante C, Haddy N, Baudin E, et al. Long-term outcome of 444 patients with distant metastases from papillary and follicular thyroid carcinoma: benefits and limits of radioiodine therapy. J Clin Endocrinol Metab. 2006;91(8):2892-2899.

8. Sherman SI. Cytotoxic chemotherapy for differentiated thyroid carcinoma. Clin Oncol (R Coll Radiol). 2010;22(6):464-468.

9. Wells SA Jr, Robinson BG, Gagel RF, et al. Vandetanib in patients with locally advanced or metastatic medullary thyroid cancer: a randomized, double-blind Phase III trial. J Clin Oncol. 2012;30(2):134-141.

10. Elisei R, Schlumberger MJ, Müller SP, et al. Cabozantinib in progressive medullary thyroid cancer. J Clin Oncol. 2013;31(29):3639-3646.

11. Pawson T. Regulation and targets of receptor tyrosine kinases. Eur $J$ Cancer. 2002;38 Suppl 5:S3-S10.

12. Nikiforova MN, Nikiforov YE. Molecular genetics of thyroid cancer: implications for diagnosis, treatment and prognosis. Expert Rev Mol Diagn. 2008;8(1):83-95.

13. Ricarte-Filho JC, Ryder M, Chitale DA, et al. Mutational profile of advanced primary and metastatic radioactive iodine-refractory thyroid cancers reveals distinct pathogenetic roles for BRAF, PIK3CA, and AKT1. Cancer Res. 2009;69(11):4885-4893.

14. Krause DS, Van Etten RA. Tyrosine kinases as targets for cancer therapy. N Engl J Med. 2005;353(2):172-187.

15. Antonelli A, Fallahi P, Ferrari SM, et al. Dedifferentiated thyroid cancer: a therapeutic challenge. Biomed Pharmacother. 2008;62(8):559-563.

16. Nikiforov YE. Thyroid carcinoma: molecular pathways and therapeutic targets. Mod Pathol. 2008;21 Supp1 2:S37-S43.

17. Kroll TG, Sarraf P, Pecciarini L, et al. PAX8-PPARgamma1 fusion oncogene in human thyroid carcinoma [corrected]. Science. 2000;289(5483):1357-1360.

18. Hou P, Liu D, Xing M. Functional characterization of the T17991801del and A1799-1816ins BRAF mutations in papillary thyroid cancer. Cell Cycle. 2007;6(3):377-379.

19. Saji M, Ringel MD. The PI3K-Akt-mTOR pathway in initiation and progression of thyroid tumors. Mol Cell Endocrinol. 2010;321(1):20-28.
20. Mulligan LM, Kwok JB, Healey CS, et al. Germ-line mutations of the RET proto-oncogene in multiple endocrine neoplasia type $2 \mathrm{~A}$. Nature. 1993;363(6428):458-460

21. Learoyd DL, Messina M, Zedenius J, Robinson BG. Molecular genetics of thyroid tumors and surgical decision-making. World J Surg. 2000;24(8):923-933.

22. Holbro T, Civenni G, Hynes NE. The ErbB receptors and their role in cancer progression. Exp Cell Res. 2003;284(1):99-110.

23. Rodríguez-Antona C, Pallares J, Montero-Conde C, et al. Overexpression and activation of EGFR and VEGFR2 in medullary thyroid carcinomas is related to metastasis. Endocr Relat Cancer. 2010;17(1):7-16.

24. Bottaro DP, Rubin JS, Faletto DL, et al. Identification of the hepatocyte growth factor receptor as the c-met proto-oncogene product. Science. 1991;251(4995):802-804.

25. Klein M, Picard E, Vignaud JM, et al. Vascular endothelial growth factor gene and protein: strong expression in thyroiditis and thyroid carcinoma. J Endocrinol. 1999;161(1):41-49.

26. Bunone G, Vigneri P, Mariani L, et al. Expression of angiogenesis stimulators and inhibitors in human thyroid tumors and correlation with clinical pathological features. Am J Pathol. 1999;155(6): 1967-1976.

27. Kerbel RS. Tumor angiogenesis. $N$ Engl J Med. 2008;358(19): 2039-2049.

28. Eggo MC, Hopkins JM, Franklyn JA, Johnson GD, Sanders DS, Sheppard MC. Expression of fibroblast growth factors in thyroid cancer. J Clin Endocrinol Metab. 1995;80(3):1006-1011.

29. Wesche J, Haglund K, Haugsten EM. Fibroblast growth factors and their receptors in cancer. Biochem J. 2011;437(2):199-213.

30. Haugsten EM, Wiedlocha A, Olsnes S, Wesche J. Roles of fibroblast growth factor receptors in carcinogenesis. Mol Cancer Res. 2010;8(11): 1439-1452.

31. St Bernard R, Zheng L, Liu W, Winer D, Asa SL, Ezzat S. Fibroblast growth factor receptors as molecular targets in thyroid carcinoma. Endocrinology. 2005;146(3):1145-1153.

32. Bergers G, Hanahan D. Modes of resistance to anti-angiogenic therapy. Nat Rev Cancer. 2008;8(8):592-603.

33. Dieci MV, Arnedos M, Andre F, Soria JC. Fibroblast growth factor receptor inhibitors as a cancer treatment: from a biologic rationale to medical perspectives. Cancer Discov. 2013;3(3):264-279.

34. Polverino A, Coxon A, Starnes C, et al. AMG 706, an oral, multikinase inhibitor that selectively targets vascular endothelial growth factor, platelet-derived growth factor, and kit receptors, potently inhibits angiogenesis and induces regression in tumor xenografts. Cancer Res. 2006;66(17):8715-8721.

35. Sherman SI, Wirth LJ, Droz JP, et al. Motesanib diphosphate in progressive differentiated thyroid cancer. $N$ Engl J Med. 2008;359(1): $31-42$.

36. Schlumberger MJ, Elisei R, Bastholt L, et al. Phase II study of safety and efficacy of motesanib in patients with progressive or symptomatic, advanced or metastatic medullary thyroid cancer. J Clin Oncol. 2009; 27(23):3794-3801.

37. Cohen EE, Rosen LS, Vokes EE, et al. Axitinib is an active treatment for all histologic subtypes of advanced thyroid cancer: results from a Phase II study. J Clin Oncol. 2008;26(29):4708-4713.

38. Locati L, Licitra L, Agate I, et al. Phase 2 trial of axitinib for advanced thyroid cancer: preliminary activity results. Presented at the Fifth European Conference on Head and Neck Oncology, Poznan, Poland, April 18-21, 2012.

39. Kloos RT, Ringel MD, Knopp MV, et al. Phase II trial of sorafenib in metastatic thyroid cancer. J Clin Oncol. 2009;27(10):1675-1684.

40. Gupta-Abramson V, Troxel AB, Nellore A, et al. Phase II trial of sorafenib in advanced thyroid cancer. J Clin Oncol. 2008;26(29): 4714-4719.

41. Hoftijzer H, Heemstra KA, Morreau H, et al. Beneficial effects of sorafenib on tumor progression, but not on radioiodine uptake, in patients with differentiated thyroid carcinoma. Eur J Endocrinol. 2009; 161(6):923-931. 
42. Ahmed M, Barbachano Y, Riddell A, et al. Analysis of the efficacy and toxicity of sorafenib in thyroid cancer: a Phase II study in a UK based population. Eur J Endocrinol. 2011;165(2):315-322.

43. Brose MS, Nutting C, Jarzab B, et al. Sorafenib in locally advanced or metastatic patients with radioactive iodine-refractory differentiated thyroid cancer: the Phase III DECISION trial. J Clin Oncol. 2013; 31 Suppl:Abstr 4.

44. Lam ET, Ringel MD, Kloos RT, et al. Phase II clinical trial of sorafenib in metastatic medullary thyroid cancer. J Clin Oncol. 2010;28(14): 2323-2330.

45. Capdevila J, Iglesias L, Halperin I, et al. Sorafenib in metastatic thyroid cancer. Endocr Relat Cancer. 2012;19(2):209-216.

46. Cohen EE, Needles BM, Cullen KJ, et al. Phase 2 study of sunitinib in refractory thyroid cancer. J Clin Oncol. 2008;26 Suppl:Abstr 6025.

47. Ravaud A, Fouchardière C, Courbon F, et al. Sunitinib in patients with refractory advanced thyroid cancer: the THYSU Phase II trial. J Clin Oncol. 2008;26 Suppl:Abstr 6058.

48. Carr LL, Mankoff DA, Goulart BH, et al. Phase II study of daily sunitinib in FDG-PET-positive, iodine-refractory differentiated thyroid cancer and metastatic medullary carcinoma of the thyroid with functional imaging correlation. Clin Cancer Res. 2010;16(21):5260-5268.

49. De Souza JA, Busaidy N, Zimrin A, et al. Phase II trial of sunitinib in medullary thyroid cancer (MTC). J Clin Oncol. 2010;15 Suppl:Abstr 5504.

50. Bible KC, Suman VJ, Molina JR, et al. Efficacy of pazopanib in progressive, radioiodine-refractory, metastatic differentiated thyroid cancers: results of a Phase 2 consortium study. Lancet Oncol. 2010;11(10): 962-972.

51. Bible KC, Suman VJ, Menefee ME, et al. A multiinstitutional Phase 2 trial of pazopanib monotherapy in advanced anaplastic thyroid cancer. J Clin Endocrinol Metab. 2012;97(9):3179-3184.

52. Wells SA Jr, Gosnell JE, Gagel RF, et al. Vandetanib for the treatment of patients with locally advanced or metastatic hereditary medullary thyroid cancer. J Clin Oncol. 2010;28(5):767-772.
53. Robinson BG, Paz-Ares L, Krebs A, Vasselli J, Haddad R. Vandetanib (100 mg) in patients with locally advanced or metastatic hereditary medullary thyroid cancer. J Clin Endocrinol Metab. 2010;95(6): 2664-2671.

54. Leboulleux S, Bastholt L, Krause T, et al. Vandetanib in locally advanced or metastatic differentiated thyroid cancer: a randomised, double-blind, Phase 2 trial. Lancet Oncol. 2012;13(9):897-905.

55. Kurzrock R, Sherman SI, Ball DW, et al. Activity of XL184 (cabozantinib), an oral tyrosine kinase inhibitor, in patients with medullary thyroid cancer. J Clin Oncol. 2011;29(19):2660-2666.

56. Matsui J, Funahashi Y, Uenaka T, Watanabe T, Tsuruoka A, Asada M. Multi-kinase inhibitor E7080 suppresses lymph node and lung metastases of human mammary breast tumor MDA-MB-231 via inhibition of vascular endothelial growth factor-receptor (VEGF-R) 2 and VEGF-R3 kinase. Clin Cancer Res. 2008;14(17):5459-5465.

57. Grande E, Diez JJ, Zafon C, Capdevila J. Thyroid cancer: molecular aspects and new therapeutic strategies. J Thyroid Res. 2012;2012: 847108.

58. Boss DS, Glen H, Beijnen JH, et al. A Phase I study of E7080, a multitargeted tyrosine kinase inhibitor, in patients with advanced solid tumours. Br J Cancer. 2012;106(10):1598-1604.

59. Sherman SI, Jarzab B, Cabanillas ME, et al. A Phase II trial of the multitargeted kinase inhibitor E7080 in advanced radioiodine (RAI)refractory differentiated thyroid cancer (DTC). J Clin Oncol. 2011; 29 Suppl:Abstr 5503.

60. Ball DW, Sherman SI, Jarzab B, et al. Lenvatinib treatment of advanced RAI-refractory differentiated thyroid cancer (DTC): cytokine and angiogenic factor $(\mathrm{CAF})$ profiling in combination with tumor genetic analysis to identify markers associated with response. J Clin Oncol. 2012;30 Suppl:Abstr 5518.

61. Schlumberger M, Jarzab B, Cabanillas ME, et al. A Phase II trial of the multitargeted kinase inhibitor lenvatinib (E7080) in advanced medullary thyroid cancer (MTC). J Clin Oncol. 2012;30 Suppl:Abstr 5591.
Biologics: Targets \& Therapy

\section{Publish your work in this journal}

Biologics: Targets \& Therapy is an international, peer-reviewed journal focusing on the patho-physiological rationale for and clinical application of Biologic agents in the management of autoimmune diseases, cancers or other pathologies where a molecular target can be identified. This journal is indexed on PubMed Central, CAS, EMBase, Scopus

\section{Dovepress}

and the Elsevier Bibliographic databases. The manuscript management system is completely online and includes a very quick and fair peerreview system, which is all easy to use. Visit http://www.dovepress. com/testimonials.php to read real quotes from published authors. 